\title{
Dietary Exposure to Heavy Metals via Consumption of Some Vegetables and Parts of Cow Meat by Adults in Awka, Nigeria.
}

\author{
V. I Onwukeme ${ }^{1}$ P. M Nwako ${ }^{2}$ M. N. Mgbemena3and M. U. Onuoha ${ }^{4}$ \\ ${ }^{1,2,4}$ department Of Pure \& Industrial Chemistry, Nnamdi Azikiwe University, Awka, Nigeria. \\ ${ }^{3}$ department Of Chemistry, Federal University Of Agriculture,Umudike,Abia State,Nigeria
}

\begin{abstract}
Dietary Exposure of adult population in Awka, Nigeria to toxic/heavy metal was carried out using analysis of some Vegetable and parts of cow meat samples commonly consumed in the study area, as well as food frequency questionnaire. The results showed that Lead $(\mathrm{Pb})$ and Cadmium $(\mathrm{Cd})$ were the risk factors to adult population in the area based on Provisional Tolerable Daily Intake (PTDI) for toxic metals and Acceptable Daily Intake (ADI) for other metals. It was observed that while estimated provisional daily intake of these metal in all vegetable samples fell within the limit set by European Commission, only cent leave was found to contribute significant amount of lead $(\mathrm{Pb})$ to adult population in the area. Similarly, meat samples especially cow intestine and kidney consumption were the major contributors of Cadmium $(\mathrm{Cd})$ to adult population in the area .This calls for precautionary measures in the consumption of these products especially meat samples due to their adverse health implications.
\end{abstract}

Key Words: Toxic/heavy metals, Vegetables and Meat samples, risk assessment.

\section{Introduction}

Industrialization, agriculture and poor planning are the major causes of heavy metal pollution in the Nigerian environment. These metals persist because they cannot be degraded in the environment [1].The pollutants from industries contain heavy metals such as Cadmium, Lead, Mercury and Arsenic which are toxic and have little or no beneficial effects on man and wildlife. Heavy metals have the ability to accumulate in living organism and at elevated levels can be toxic to the organism. They enter into the food material and ultimately make their passage into the tissue. Elevated levels of copper can cause brain damage [2].Heavy metal contamination of food can take place during handling, processing of food from the farm to the place where they are consumed [3].Apart from growth of plants in contaminated soil, feeding of animals on feeds containing these metals, storage and packaging containers, water used in cooking and preparation of beverage also contribute sufficient amount. Human contamination occurs through ingestion or inhalation. Contamination by inhalation of dust is important especially for metals that have low vapour pressure which can easily be mobilized from the soil and other components of the environment like Cadmium \& Mercury [3].In addition, reports have shown that various activities by man in recent years have increased the quantity and distribution of heavy metals in the environment. Such activity like indiscriminate dumping of agricultural waste could lead to pollution of rivers due to run-off [3].Vegetables such as Veronia amygdaline, Occium Viride, Daucus Carota and Solonium ambergine are also eaten raw. Meats are some times roasted instead of boiled before they are consumed. Heavy metals often have direct physiological toxic effect. Vegetables are important component of diet because it contributes protein, Vitamin, Iron, Calcium and other nutrients usually in short supply. They act as buffering agents for acidic substances produced during food digestion but contain essential and toxic elements over wide range of concentrations. Vegetables get toxic metals from soil, air and from polluted environment. Report shows that nearly $1 / 2$ of mean of food ingestion of lead, calcium and mercury are through plant $[3,4]$.Lead are a metabolic poison and a neurotoxin that binds to essential enzymes and several other cellular components and inactivates them. Toxic effects of lead are seen on haemopoietic, nervous, gastrointestinal and renal systems. It is estimated that $1 / 2$ of human lead intake is from food and $1 / 2$ from plant[5].Similarly, increase in levels of Copper cause liver, Kidney and brain damages, which may follow haemolytic crisis [6].These diseases, may be caused by consuming contaminated meat ,garden egg, and other foods[7].In Nigeria, Sokoto State is the largest producer of cattles [8,9].The beef production capacity of the state is currently put at 500,000 tones per annum[5,9]. As Nigeria aspires to improve the life expectancy of her citizen and become one of the 20 best economies of the world by 2020,signed by the Millennium Development Goal(MDG), the issue of food security and assessment of risk associated with consumption of a particular food substance cannot be over emphasized. W.H.O recommends 8 fruits and Vegetable per person per day [10].There is a common notion that the more vegetables consumed the better. Although vegetables have advantages such as constipation control, reduction in cholesterol level, risk of diabetes, hypertension, cancer and heart diseases. It has also been reported that some vegetables and commercially produced food are hyper- accumulators of toxic heavy metals [11].Rearing of livestock in proximity of polluted environment was reported to contribute to heavy 
Dietary Exposure to Heavy Metals via Consumption of Some Vegetables and Parts of Cow ...

metal contamination of meat product. Risk assessment involves risk identification, hazard characterization (using material regulatory acceptable standards).Exposure assessment (using contamination data and food consumption data) to obtain level of intake and risk characterization (using ADI and PTWI and TDI) to evaluate risk for the individual. It has been reported that some metals like copper accumulate more in shoot than roots of plants [13].No work has been done on dietary exposure and risk assessment of heavy metals through vegetables and some meat consumption in Awka metropolis, Anambra State Nigeria

This work is aimed at assessing dietary exposure/Intake of heavy metal contamination in some vegetables and parts of cow meat and to estimate their health risk using analysis of individual food item and food frequency questionnaire. This is because food is an important source of overall exposure and undertaking its risk assessment becomes very important too. Contribution of each food to heavy metal contamination is also of interest especially for epidemiological study of disease. ADI represents safe intake level for a healthy adult of normal body weight who consumes an average amount daily. It measures toxicity for a long time exposure of a chemical substance as oppposed to acute toxicity.

\section{Materials And Method}

Collection of Samples: The Samples were collected using hand gloves made of polyethylene. Fresh samples of cow liver, kidney and beef were collected from slaughter house called Kwata. The samples were collected with a quartz and put in a polyethylene bag, taken to the laboratory for preparation and analysis.

PREPARATION OF SAMPLES: The collected samples were washed with distilled water to obtain a wet weight. The meat samples were dried in an oven at $105^{\circ} \mathrm{C}$ for $48 \mathrm{hrs}$. The local pears were cut into pieces before drying.

\section{Preparation Of Meat Sample}

Before collecting the meat samples, a hand glove made of polyethylene material was worn. The sample was collected from Kwata slaughter in Awka North L.G.A, Anambra State. The Liver, Intestine meat and Beef (red meat) were collected. The samples were washed with distilled water to obtain a wet weight. The meat samples were dried in an oven at $105^{\circ} \mathrm{C}$ for $48 \mathrm{hrs}$.the samples were ground into a fine powder using manual grinder (Coroner Model) and then taken to the laboratory for digestion.

\section{Preparation Of Vegetable Sample}

The collected vegetable samples were washed with distilled water to obtain a wet weight .The samples were dried in an oven at $105^{\circ} \mathrm{C}$ for $48 \mathrm{hrs}$. They were ground into a fine powder using Coroner blender and then taken to the laboratory for digestion.

\section{Digestion Of Meat Sample}

$1 \mathrm{~g}$ of dried meat sample was digested with $10 \mathrm{~cm}^{3}$ of $1: 4 \mathrm{HNO}_{3}: \mathrm{H}_{2} \mathrm{O}_{2}$ acid mixture using a mino Kjeldahl vessel in a fume cupboard at $120^{\circ} \mathrm{C}$ for $1 \mathrm{hr}$.The digest was filtered and diluted with $50 \mathrm{~cm}^{3}$ of distilled water. The solution was analyzed with AAS 200fs.

\section{Digestion Of Vegetable /Food Sample}

$1 \mathrm{~g}$ of each sample was were and added into a $10 \mathrm{~cm}^{3}$ of aqua rajia and heated with a hotplate in fume cupboard for 20min.After complete digestion of the sample ,the flask was allowed to cool and then $10 \mathrm{~cm}^{3}$ distilled water was added to the digested samples and the solution filtered. The filtrate was made up to $50 \mathrm{~cm}^{3}$ with distilled water and kept for AAS analysis

RESULTS AND DISCUSSION: The results of these analysis are presented in tables 1 and 3 and estimated daily Intake in Tables 2 and 4

TABLE 1.0: Concentration of Heavy Metals in Vegetable Food mg/kg

\begin{tabular}{|c|c|c|c|c|c|c|c|c|}
\hline Vegetable/Metal & Botanical Name & $\mathrm{Pb}$ & $\mathrm{Cu}$ & $\mathrm{Co}$ & $\mathrm{Zn}$ & As & $\mathrm{Cd}$ & $\mathrm{Cr}$ \\
\hline Carrot & Daucas carota & $0.16 \pm 4.1$ & $0.43 \pm 0.01$ & $\begin{array}{l}1.06 \pm \\
0.03\end{array}$ & $\begin{array}{l}0.34 \pm \\
0.007\end{array}$ & $\begin{array}{l}0.96 \pm \\
0.05\end{array}$ & $\begin{array}{l}0.13 \pm \\
0.04\end{array}$ & $\begin{array}{l}0.22 \pm \\
0.21\end{array}$ \\
\hline Garden Egg & Solamom gilo & $1.31 \pm 0.02$ & $0.09 \pm 0.11$ & $\begin{array}{l}0.69 \pm \\
0.003 \\
\end{array}$ & $\begin{array}{l}1.23 \pm \\
0.01\end{array}$ & $\begin{array}{l}0.02 \pm \\
0.10 \\
\end{array}$ & $\begin{array}{l}0.23 \pm \\
0.005 \\
\end{array}$ & $0.43 \pm 0.1$ \\
\hline Local pear & Dacyodes caules & $0.30 \pm 0.02$ & $0.56 \pm 0.20$ & $\begin{array}{l}0.24 \pm \\
0.001 \\
\end{array}$ & $\begin{array}{l}0.36 \pm \\
0.0005\end{array}$ & $\begin{array}{l}0.62 \pm \\
0.14\end{array}$ & $\begin{array}{l}0.11 \pm \\
0.002\end{array}$ & $\begin{array}{l}0.14 \pm \\
0.25 \\
\end{array}$ \\
\hline Bitter leaf & $\begin{array}{l}\text { Veroma } \\
\text { amyamine }\end{array}$ & $1.25 \pm 0.30$ & $1.90 \pm 0.45$ & $\begin{array}{l}2.00 \pm \\
0.14 \\
\end{array}$ & $\begin{array}{l}0.69 \pm \\
0.001 \\
\end{array}$ & $\begin{array}{l}0.70 \pm \\
0.09 \\
\end{array}$ & $\begin{array}{l}0.23 \pm \\
0.19 \\
\end{array}$ & $\begin{array}{l}0.21 \pm \\
0.31 \\
\end{array}$ \\
\hline $\begin{array}{l}\begin{array}{l}\text { Tobacco leaf } \\
\text { (Snuff) }\end{array} \\
\end{array}$ & $\begin{array}{l}\text { Nicotoana } \\
\text { tobacum }\end{array}$ & 0.0001 & $\begin{array}{l}0.001 \pm \\
0.001\end{array}$ & $\begin{array}{l}0.10 \pm \\
0.01\end{array}$ & $\begin{array}{l}3.60 \pm \\
0.003\end{array}$ & 0.001 & BDL & $\begin{array}{l}0.10 \pm \\
0.22 \\
\end{array}$ \\
\hline Fodder grass & $\begin{array}{l}\text { Anciama } \\
\text { flavouroschott }\end{array}$ & $\begin{array}{l}1.01 \pm \\
0.0003\end{array}$ & $0.99 \pm 0.07$ & $\begin{array}{l}1.11 \pm \\
0.0001 \\
\end{array}$ & $\begin{array}{l}0.10 \pm \\
0.004 \\
\end{array}$ & $\begin{array}{l}0.25 \pm \\
0.10 \\
\end{array}$ & $\begin{array}{l}0.34 \pm \\
0.02 \\
\end{array}$ & $0.32 \pm 0.1$ \\
\hline
\end{tabular}


Dietary Exposure to Heavy Metals via Consumption of Some Vegetables and Parts of Cow ...

TABLE 2.0: Estimated Daily Intake of Heavy Metals in $\mu \mathrm{g} / \mathrm{kg}$ bw/day (PTDI) and (ADI)for the vegetable samples and European Commission Standard.

\begin{tabular}{|c|c|c|c|c|c|c|c|c|c|}
\hline & $\begin{array}{c}\text { Botanical } \\
\text { Name }\end{array}$ & $\begin{array}{c}\text { Pb* }^{*} \\
\mu g / k g \\
\text { bw/da } \\
y\end{array}$ & $\begin{array}{c}\mathrm{Cu} \mu \mathrm{g} / \mathrm{kg} \\
\text { ADI }\end{array}$ & $\begin{array}{c}\text { Co } \\
\mu \mathrm{g} / \mathrm{kg} \\
\text { ADI }\end{array}$ & $\begin{array}{c}\mathrm{Zn} \\
\mu \mathrm{g} / \mathrm{kg} \\
\mathrm{ADI}\end{array}$ & $\begin{array}{c}\text { As* } \\
\mu \mathrm{g} / \mathrm{kg} \\
\mathrm{bw} / \mathrm{d} \\
\text { ay }\end{array}$ & $\begin{array}{c}\text { Cd* } \\
\mu g / k g \\
\text { bw/day }\end{array}$ & $\begin{array}{c}\mathrm{Cr} \\
\mu \mathrm{g} / \mathrm{kg} \\
/ \mathrm{day}\end{array}$ & $\begin{array}{c}\text { Av. } \\
\text { consumptio } \\
n \text { data in } \\
\mu \mathrm{g} / \text { pesson/d } \\
\text { ay (dry wt) }\end{array}$ \\
\hline Carrot & $\begin{array}{l}\text { Daucas carota } \\
\mu \mathrm{g} / \text { person/day } \\
\mu \mathrm{g} / \mathrm{kg} \text { bw/day }\end{array}$ & 0.105 & $\begin{array}{l}12.60 \\
0.21 \\
\end{array}$ & $\begin{array}{l}42.00 \\
0.70 \\
\end{array}$ & $\begin{array}{l}13.80 \\
0.23 \\
\end{array}$ & 0.64 & 0.08 & 0.15 & $6.70 \mathrm{~g}$ \\
\hline $\begin{array}{l}\text { Garden } \\
\text { egg }\end{array}$ & $\begin{array}{l}\text { Solamom gilo } \\
\mu \mathrm{g} / \text { person/day } \\
\mu \mathrm{g} / \text { person/day }\end{array}$ & 1.78 & $\begin{array}{l}7.20 \\
0.12 \\
\end{array}$ & $\begin{array}{l}55.20 \\
0.92 \\
\end{array}$ & $\begin{array}{l}99.00 \\
1.65 \\
\end{array}$ & 0.02 & 0.30 & 0.57 & $80.83 \mathrm{~g}$ \\
\hline $\begin{array}{l}\text { Local } \\
\text { pear }\end{array}$ & $\begin{array}{l}\text { Dacyodes } \\
\text { caules } \\
\mu \mathrm{g} / \text { person/day } \\
\mu \mathrm{g} / \mathrm{kg} \text { bw/day }\end{array}$ & 0.18 & $\begin{array}{l}19.80 \\
0.33\end{array}$ & $\begin{array}{l}8.40 \\
0.14\end{array}$ & $\begin{array}{l}12.60 \\
0.21\end{array}$ & 0.37 & 0.06 & $\begin{array}{l}4.80 \\
0.08\end{array}$ & $36.22 \mathrm{~g}$ \\
\hline $\begin{array}{l}\text { Bitter } \\
\text { leaf }\end{array}$ & $\begin{array}{l}\text { Veroma } \\
\text { amyamine } \\
\mu \text { gperson/day } \\
\mu \mathrm{g} / \mathrm{kg} \text { bw/day }\end{array}$ & 1.88 & $\begin{array}{l}172.20 \\
2.87\end{array}$ & $\begin{array}{l}1.26 \\
0.021\end{array}$ & $\begin{array}{l}62.40 \\
1.04\end{array}$ & 1.06 & 0.35 & $\begin{array}{l}19.20 \\
0.32\end{array}$ & $90.635 \mathrm{~g}$ \\
\hline Cent leaf & $\begin{array}{l}\text { Ociom viride } \\
\mu \mathrm{g} / \text { person/day } \\
\mu \mathrm{g} / \mathrm{kg} \text { bw/day }\end{array}$ & 3.87 & $\begin{array}{l}25.20 \\
0.42\end{array}$ & $\begin{array}{l}60.60 \\
1.01\end{array}$ & $\begin{array}{l}49.80 \\
0.83\end{array}$ & 0.72 & 0.38 & $\begin{array}{l}19.08 \\
0.318\end{array}$ & $100.699 \mathrm{~g}$ \\
\hline $\begin{array}{l}\text { Tobacco } \\
\text { snuff }\end{array}$ & $\begin{array}{l}\text { Nicotoana } \\
\text { tobacum } \\
\mu \mathrm{g} / \text { person/day } \\
\mu \mathrm{g} / \mathrm{kg} \text { bw/day }\end{array}$ & 0.01 & $\begin{array}{l}468.00 \\
7.48\end{array}$ & $\begin{array}{l}4.20 \\
0.07\end{array}$ & $\begin{array}{l}101.4 \\
0 \\
2.69 \\
\end{array}$ & 0.01 & BDL & $\begin{array}{l}4.20 \\
0.07\end{array}$ & $44.80 \mathrm{~g}$ \\
\hline $\begin{array}{l}\text { Fodder } \\
\text { Grass }\end{array}$ & $\begin{array}{l}\text { Anciama } \\
\text { flavouroschott } \\
\mu \mathrm{g} / \text { person/day } \\
\mu \mathrm{g} / \mathrm{kg} \text { bw/day }\end{array}$ & - & - & - & - & - & - & - & - \\
\hline $\begin{array}{l}\text { European } \\
\text { Commiss } \\
\text { ion std } \\
\text { PTDI }\end{array}$ & & $\begin{array}{l}3.5 \\
\mu \mathrm{g} / \mathrm{kgb} \\
\text { w/day }\end{array}$ & & & & $\begin{array}{l}7.1 \\
\mu \mathrm{g} / \mathrm{kg} \\
\mathrm{bw} / \mathrm{da} \\
\mathrm{y}\end{array}$ & $\begin{array}{l}1.0 \\
\mu \mathrm{g} / \mathrm{kgb} \\
\text { w/day }\end{array}$ & & \\
\hline PTWI & & $\begin{array}{l}25 \\
\mu \mathrm{g} / \mathrm{kgb} \\
\mathrm{w} / \mathrm{wk}\end{array}$ & & $\begin{array}{l}6 \\
\mu \mathrm{g} / \mathrm{kg} \\
\mathrm{bw} / \mathrm{w} \\
\mathrm{k}\end{array}$ & & $\begin{array}{l}50 \\
\mu \mathrm{g} / \mathrm{kg} \\
\mathrm{bw} / \mathrm{w} \\
\mathrm{k} \\
\end{array}$ & $\begin{array}{l}7.0 \\
\mu \mathrm{g} / \mathrm{kgb} \\
w / w k\end{array}$ & & \\
\hline \multicolumn{10}{|l|}{ ADI std } \\
\hline $\begin{array}{l}\text { USA } \\
2009 \\
\end{array}$ & & & & & $\begin{array}{l}11 \\
\mu \mathrm{g} / \mathrm{kg}\end{array}$ & & & $\begin{array}{l}35 \\
\mu \mathrm{g} / \mathrm{kg} \\
\end{array}$ & \\
\hline $\begin{array}{l}\text { WHO } \\
2001\end{array}$ & & & $\begin{array}{l}900- \\
10000 \\
\mu \mathrm{g} / \mathrm{KG} \\
\end{array}$ & $\begin{array}{l}3.0 \\
\mu \mathrm{g} / \mathrm{K} \\
\mathrm{G} \\
\end{array}$ & & & & & \\
\hline $\begin{array}{l}\text { European } \\
\text { Commiss } \\
\text { ion std }\end{array}$ & & $\begin{array}{l}3.5 \\
\mu \mathrm{g} / \mathrm{kg}\end{array}$ & $500 \mu \mathrm{g} / \mathrm{kg}$ & $\begin{array}{l}3.0 \\
\mu \mathrm{g} / \mathrm{kg}\end{array}$ & $\begin{array}{l}11 \\
\mu \mathrm{g} / \mathrm{kg}\end{array}$ & $\begin{array}{l}7.1 \\
\mu \mathrm{g} / \mathrm{kg}\end{array}$ & $\begin{array}{l}1.0 \\
\mu \mathrm{g} / \mathrm{kg}\end{array}$ & $\begin{array}{l}35 \\
\mu \mathrm{g} / \mathrm{kg}\end{array}$ & \\
\hline
\end{tabular}

Key:

PTD1 - Provisional Tolerable Daily Intake ( $\mu \mathrm{g} / \mathrm{kgbw} /$ day)

PTW1 - Provisional Tolerable Weekly Intake $\mu \mathrm{g} / \mathrm{kgbw} / \mathrm{week}$

AD1 - Acceptable Daily Intake $(\mu \mathrm{g} / \mathrm{kg})$

TABLE 3.0: Concentration of Heavy Metals in Parts of Cow Meat Mg/Kg

\begin{tabular}{|c|c|c|c|c|c|c|c|c|}
\hline Cow Meat & $\mathrm{Pb}$ & $\mathrm{Cu}$ & $\mathrm{Co}$ & $\mathrm{Zn}$ & As & $\mathrm{Cd}$ & $\mathrm{Cr}$ & $\begin{array}{c}\text { Av. } \\
\text { consumption } \\
\text { data g/day } \\
\text { (dry wt) } \\
\end{array}$ \\
\hline Liver & $\begin{array}{l}1.31 \pm \\
0.002\end{array}$ & $\begin{array}{l}2.02 \pm \\
2.09\end{array}$ & $\begin{array}{l}0.67 \pm \\
0.80\end{array}$ & $\begin{array}{l}1.23 \pm \\
0.0005\end{array}$ & $0.06 \pm 2.0$ & $0.23 \pm 2.60$ & $\begin{array}{l}0.50 \pm \\
0.20\end{array}$ & $76.64 \mathrm{~g}$ \\
\hline Kidney & $2.40 \pm 0.10$ & $\begin{array}{l}1.08 \pm \\
0.05 \\
\end{array}$ & $\begin{array}{l}0.78 \pm \\
0.01 \\
\end{array}$ & $3.45 \pm 0.01$ & $\begin{array}{l}0.01 \pm \\
0.10 \\
\end{array}$ & $\begin{array}{l}.34 \pm \\
0.021 \\
\end{array}$ & $\begin{array}{l}1.30 \pm \\
0.001 \\
\end{array}$ & $48.32 \mathrm{~g}$ \\
\hline $\begin{array}{l}\text { Beef (Red } \\
\text { Meat) }\end{array}$ & $\begin{array}{l}1.12 \pm \\
0.024\end{array}$ & $\begin{array}{l}0.09 \pm \\
0.2\end{array}$ & $\begin{array}{l}0.44 \pm \\
0.003\end{array}$ & $\begin{array}{l}0.52 \pm \\
0.005\end{array}$ & $\begin{array}{l}0.06 \pm \\
0.01\end{array}$ & $\begin{array}{l}0.54 \pm \\
0.0003\end{array}$ & $\begin{array}{l}0.39 \pm \\
0.30\end{array}$ & $156.32 \mathrm{~g}$ \\
\hline & $\begin{array}{l}0.14 \pm \\
0.003 \\
\end{array}$ & $\begin{array}{l}0.09 \pm \\
0.12 \\
\end{array}$ & $\begin{array}{l}0.76 \pm \\
0.002 \\
\end{array}$ & $0.45 \pm 0.02$ & $0.01 \pm 0.0$ & $\begin{array}{l}1.34 \pm \\
0.001 \\
\end{array}$ & $\begin{array}{l}0.14 \pm \\
0.21 \\
\end{array}$ & $140.29 \mathrm{~g}$ \\
\hline
\end{tabular}


Dietary Exposure to Heavy Metals via Consumption of Some Vegetables and Parts of Cow ...

TABLE 4.0: Estimated Daily Intake of Heavy Metals for the Parts of cow meat samples and European Commission Standard in $\mu \mathrm{g} / \mathrm{kg}$ bw/day.

\begin{tabular}{|c|c|c|c|c|c|c|c|}
\hline Sample & $\mathrm{Pb}$ & $\mathrm{Cu}$ & Co & $\mathrm{Zn}$ & As & $\mathrm{Cd}$ & $\mathrm{Cr}$ \\
\hline $\begin{array}{l}\text { Cow Liver (CL) } \\
\mu \mathrm{g} / \text { person/day } \\
\mu \mathrm{g} / \mathrm{kgbw} / \mathrm{day}\end{array}$ & 1.67 & $\begin{array}{l}154.80 \\
2.58\end{array}$ & $\begin{array}{l}51.00 \\
0.85\end{array}$ & $\begin{array}{l}157.20 \\
2.62\end{array}$ & 0.07 & 0.29 & $\begin{array}{l}37.80 \\
0.63\end{array}$ \\
\hline $\begin{array}{l}\text { Cow Kidney } \\
\text { (CK) } \\
\mu \mathrm{g} / \text { person/day } \\
\mu \mathrm{g} / \mathrm{kgbw} / \text { day }\end{array}$ & 1.61 & $\begin{array}{l}51.60 \\
0.86\end{array}$ & $\begin{array}{l}37.20 \\
0.62\end{array}$ & $\begin{array}{l}166.20 \\
2.77\end{array}$ & 0.05 & 3.49 & $\begin{array}{l}62.40 \\
1.04\end{array}$ \\
\hline $\begin{array}{l}\text { Cow Beef (CB) } \\
\mu \mathrm{g} / \mathrm{person} / \mathrm{day} \\
\mu \mathrm{g} / \mathrm{kgbw} / \mathrm{day}\end{array}$ & 2.43 & $\begin{array}{l}14.40 \\
0.24\end{array}$ & $\begin{array}{l}72.60 \\
1.21\end{array}$ & $\begin{array}{l}86.40 \\
1.44\end{array}$ & 0.16 & 1.49 & $\begin{array}{l}64.80 \\
1.08\end{array}$ \\
\hline $\begin{array}{l}\text { Cow Intestine } \\
(\mathrm{CI}) \\
\mu \mathrm{g} / \text { person/day } \\
\mu \mathrm{g} / \mathrm{kgbw} / \text { day }\end{array}$ & 0.32 & $\begin{array}{l}12.60 \\
0.21\end{array}$ & $\begin{array}{l}106.20 \\
1.77\end{array}$ & $\begin{array}{l}63.00 \\
1.05\end{array}$ & 0.23 & 3.13 & $\begin{array}{l}19.20 \\
0.32\end{array}$ \\
\hline $\begin{array}{l}\text { European } \\
\text { commission } \\
\text { Std PTDI } \\
\text { PTWI }\end{array}$ & $\begin{array}{l}3.5 \mu \mathrm{g} / \mathrm{k} \\
\mathrm{g} \\
25 \mu \mathrm{g} / \mathrm{k} \\
\mathrm{g} \\
\mathrm{bw} / \mathrm{wk}\end{array}$ & & & & $\begin{array}{l}7.5 \mu \mathrm{g} / \mathrm{kgb} \\
\text { w/day } \\
50 \mu \mathrm{g} \mu \mathrm{g} / \mathrm{kg} \\
\mathrm{bw} / \mathrm{wk}\end{array}$ & $\begin{array}{l}1.0 \mu \mathrm{g} / \\
\mathrm{kg} \\
7.0 \mu \mathrm{g} / \\
\mathrm{kg}\end{array}$ & \\
\hline $\begin{array}{l}\text { USA } 2009 \text { Std } \\
\text { AD1 }\end{array}$ & & & & $11 \mathrm{mg} / \mathrm{kg}$ & $\begin{array}{l}\text { (Inorganic } \\
\text { Ar) }\end{array}$ & & $\begin{array}{l}35 \mu \mathrm{g} / \mathrm{k} \\
\mathrm{g}\end{array}$ \\
\hline $\begin{array}{l}\text { WHO } 2001 \\
\text { AD1 }\end{array}$ & & $\begin{array}{l}900- \\
10000 \mu \mathrm{g} / \\
\mathrm{kg}\end{array}$ & $3 \mu \mathrm{g} / \mathrm{kg}$ & & & & \\
\hline $\begin{array}{l}\text { European } \\
\text { Commission std } \\
\text { ADI }\end{array}$ & $\begin{array}{l}3.5 \mu \mathrm{g} / \mathrm{k} \\
\mathrm{g}\end{array}$ & $500 \mu \mathrm{g} / \mathrm{kg}$ & $3 \mu \mathrm{g} / \mathrm{kg}$ & $11 \mu \mathrm{g} / \mathrm{kg}$ & $7.1 \mu \mathrm{g} / \mathrm{kg}$ & $\begin{array}{l}1.0 \mu \mathrm{g} / \\
\mathrm{kg}\end{array}$ & $\begin{array}{l}35 \mu \mathrm{g} / \mathrm{k} \\
\mathrm{g}\end{array}$ \\
\hline
\end{tabular}

Key:

PTD1 - Provisional Tolerable Daily Intake ( $\mu \mathrm{g} / \mathrm{kgbw} / \mathrm{day})$

PTW1 - Provisional Tolerable Weekly Intake $\mu \mathrm{g} / \mathrm{kgbw} /$ week

AD1 - Acceptable Daily Intake $(\mu \mathrm{g} / \mathrm{kg})$

Calculations:

Exposure $=$ contaminant data $*$ consumption data.

Intake $\mu \mathrm{g} / \mathrm{person} / \mathrm{day}=$ conc in $\mu \mathrm{g} / \mathrm{kg} *$ consumption

$$
\text { Data in g/person/day }
$$

$$
\begin{gathered}
\frac{\text { Intake in } \mu \mathrm{g} / \mathrm{person} / \mathrm{day}}{60 \text { yrs Adult of } 60 \mathrm{~kg}}=\text { Intake } \mu \mathrm{g} / \mathrm{kg} \mathrm{bw} / \mathrm{day} \\
\text { \% ADI or \% PTWI }=\begin{array}{c}
\text { PTDI or ADI } \\
\text { PTDI cal } * 100
\end{array}
\end{gathered}
$$

OR

$$
\frac{\mathrm{ADI} \mathrm{cal} *}{\mathrm{ADI} \mathrm{std}} \frac{100}{1}
$$

Apart from Occium Viride which exceeded the provisional tolerable weekly intake for $\mathrm{Pb}$ by $110.5 \%$, all other Vegetable samples were within the tolerable limit by JECTA [5].The concentration of $\mathrm{Pb}$ in all the meat sample were within the PTWI. The concentration of $\mathrm{Pb}$ in all the vegetable samples were below the PTDI limit of $3.5 \mu \mathrm{g} / \mathrm{kg}$ bw /day except for cent leaves (Ocium Viride).This showed that cent leaf was the major contributor of $\mathrm{Pb}$ to adult population in the area. The low concentration of $\mathrm{Pb}$ in parts of meat fell in agreement with the result of this metal in fodder samples on which cows feed. This implied that fodder grass was not the 
source of lead contamination of cow meat as was observed by some authors. Excess accumulation of $\mathrm{Pb}$ in the body causes problem in the synthesis of hemoglobin, Kidney and Liver function as well as reproductive system of human[14].Also typical disease such as lead intoxication, encephalopathy, radical nerve paralysis and Saturime colic are common[12].

The concentration of copper ranged from $0.01 \pm 0.001 \mathrm{mg} / \mathrm{kg}$ in tobacco snuff to $1.90 \pm 0.45 \mathrm{mg} / \mathrm{Kg}$ in bitter leaf for the vegetable samples and $0.08 \pm 0.05 \mathrm{mg} / \mathrm{kg}$ to $2.02 \pm 2.0 \mathrm{mg} / \mathrm{kg}$ for meat sample

The estimated daily intake ranged from $12.60 \mu \mathrm{g} / \mathrm{person} / \mathrm{day}(0.21 \mu \mathrm{g} / \mathrm{kg} \mathrm{bw} /$ day $)$ in intestine to $154.80 \mu \mathrm{g} /$ person/day $(2.58 \mu \mathrm{g} / \mathrm{kg}$ bw/day) in Cow liver for the cow meat samples, $7.20 \mu \mathrm{g} / \mathrm{person} / \mathrm{day}$ $(0.12 \mu \mathrm{g} / \mathrm{kg}$ bw/day) in garden egg to $468.00 \mu \mathrm{g} / \mathrm{person} /$ day $(7.48 \mu \mathrm{g} / \mathrm{kg}$ bw/day) in tobacco snuff for the vegetable sample and $197.40 \mu \mathrm{g} /$ person/day $(0.21 \mu \mathrm{g} / \mathrm{kg}$ bw/day) in cow intestine to $2.58 \mu \mathrm{g} / \mathrm{kg}$ bw $/ \mathrm{day}$ in cow liver for the meat sample. The implication is that there is no danger in copper consumption from these food substances as the levels obtained fell below the limit set by USRRA European Commission and WHO [5].

The concentration of Cobalt ranged from $0.10 \pm 0.01$ in tobacco Snuff to $2.00 \pm 0.14 \mu \mathrm{g} / \mathrm{kg}$ in bitter leaf for the vegetable samples, $0.44 \pm 0.80 \mu \mathrm{g} / \mathrm{kg}$ in Cow beef to $0.78 \pm 0.01 \mu \mathrm{g} / \mathrm{kg}$ in cow kidney for parts of cow meat. The estimated daily intake ranged from $1.26 \mu \mathrm{g} / \mathrm{kg}(0.02 \mu \mathrm{g} / \mathrm{kg}$ bw/day) in Veronica amygdalina (bitter leaf) to $1.01 \mu \mathrm{g} / \mathrm{kg} \mathrm{bw} / \mathrm{day}$ in Occium Viride (cent leaf) for the vegetable samples, $0.62 \mu \mathrm{g} / \mathrm{kg} / \mathrm{day}$ in cow kidney to $1.77 \mu \mathrm{g} / \mathrm{kg}$ bw/day in cow intestine for the parts of cow meat.

The concentration of zinc ranged from $0.10 \pm 0.004 \mathrm{mg} / \mathrm{kg}$ in fodder grass to $3.60 \pm 0.003 \mathrm{mg} / \mathrm{kg}$ in tobacco snuff for the vegetable sample, $0.45 \pm 0.02 \mathrm{mg} / \mathrm{kg}$ in cow intestine to $3.45 \pm 0.01 \mathrm{mg} / \mathrm{kg}$ in kidney for the parts of Cow meat sample.

The estimated average daily intake ranged from $0.21 \mu \mathrm{g} / \mathrm{kg}$ bw/day in local Pear to $2.69 \mu \mathrm{g} / \mathrm{kg}$ bw $/$ day in tobacco snuff for the vegetable sample in Cow Intestine to $2.77 \mu \mathrm{g} / \mathrm{kg}$ bw/day in cow Kidney for the parts of cow meat sample.

The estimated daily intakes of zinc in all the samples studied were well below the acceptable level of $11.00 \mu \mathrm{g} / \mathrm{kg} /$ day. Zinc is an essential trace metal which acts as a cofactor in metalcoe enzyme but high concentration if zinc in human has been reported to damaged pancreas; disrupt protein synthesis [5]. However, $\mathrm{Zn}$ consumption from the selected food does not portends any inherent danger for the people in Awka, Nigeria.

The Concentration of arsenic ranged from $0.001 \pm 0.01$ in tobacco snuff to $0.96 \pm 0.051 \mu \mathrm{g} / \mathrm{kg}$ in carrot for the vegetable samples, $0.01 \pm 0.00 \mathrm{mg} / \mathrm{kg}$ in cow intestine to $0.06 \pm 0.20 \mathrm{mg} / \mathrm{kg}$ in cow liver for the parts of cow meat sample.

The estimated daily intake for arsenic ranged from $0.01 \mu \mathrm{g} / \mathrm{kg}$ bw $/$ day in tobacco snuff to $1.06 \mu 7 \mathrm{~g} / \mathrm{kg}$ bw/day in bitter leaf for the vegetable sample, $0.05 \mu \mathrm{g} / \mathrm{kg}$ bw/day in cow kidney to $0.23 \mu \mathrm{g} / \mathrm{kg}$ bw $/ \mathrm{day}$ in cow intestine for the parts of cow meat sample. The estimated provisional tolerable daily intake arseenic fell within the tolerable limit by European standard, WHO standard of $7.1 \mu \mathrm{g} / \mathrm{kg}$ bw/day. The percentage provisional tolerable limit by weekly intake(\% PTWl) of arsenic in all food sample studied were within the range from 0.1$14.9 \%$ in tobacco snuff to Bitter leaf (vegetable). Acute arsenic exposure can give symptoms such as heartache, nausea and severe gastrointestinal irritation [17].

The concentration of Cd ranged from below detectable limit (BDL) in tobacco snuff to $0.34 \mu \mathrm{g} / \mathrm{kg}$ in fodder grass for the vegetable sample, $0.23 \pm 2.60 \mu \mathrm{g} / \mathrm{kg}$ in cow liver to $4.34 \pm 0.02 \mu \mathrm{g} / \mathrm{kg}$ in kidney for the part of cow sample.

The estimated daily intake of cadmium ranged from below detectable limit (BDL) in tobacco snuff to $0.386 \mu \mathrm{g} / \mathrm{kg}$ bw $/$ day in Ocium viride for the vegetable sample, $0.29 \mu \mathrm{g} / \mathrm{kg}$ bw/day in cow liver to $3.49 \mu \mathrm{g} / \mathrm{kg}$ bw/day in cow kidney for the parts of cow meat.

The concentration of cadmium from parts of cow meat exceeded the provisional daily intake except for cow liver. However, Cd concentration in vegetable samples was below the provisional tolerable daily intake. The percentage provisional tolerable weekly intake ranges from $6.0 \%$ to $28 \%$ of PTWI for the vegetable samples, $29 \%$ to $34 \%$ for the parts of cow meat. Excess accumulation of $\mathrm{Cd}$ in the body causes lung cancer, pulmonary adenocarcinomes, prostatic and proliferative lessons, kidney dysfunction and hypertension.

The implication is that care must be taken in consumption of some parts of cow meat as due to adverse effect of exposure of Cd since they exceeded the safe limit of 30\% PTWI for toxic metals that biaccumulate in human while the consumption of vegetable $s$ have no dangerous effect as they fell below the provisional tolerable daily intake.

The concentration of chromium ranged from $0.10 \pm 0.22 \mathrm{mg} / \mathrm{kg}$ in tobacco snuff to $0.32 \pm 0.1 \mathrm{mg} / \mathrm{kg}$ in fodder grass for the vegetable samples, $0.14 \pm 0.21 \mathrm{mg} / \mathrm{kg}$ in cow intestine to $1.30 \pm 0.0 \mathrm{mg} / \mathrm{kg}$ in cow kidney for the parts cow meat sample.

The estimated daily intake of chromium also ranged from $0.07 \mu \mathrm{g} / \mathrm{kg}$ bw/day in Tobacco snuff to $0.57 \mu \mathrm{g} / \mathrm{kg}$ bw/ day in garden egg for the vegetable samples, $0.32 \mu \mathrm{g} / \mathrm{kg}$ bw $/$ day in cow intestine to $1.08 \mu \mathrm{g} / \mathrm{kg}$ bw/day in cow beef for the parts of cow meat samples. The results show that all the vegetable samples studies are within the acceptable daily intake of $35 \mu \mathrm{g} / \mathrm{kg}$ [5] while that of parts cow meat exceeded the level. The 
consumption of these parts of cow meat are the major source of contamination of Awka people Nigeria, since intake from these substances well exceeded the acceptable daily intake of $35 \mu \mathrm{g} / \mathrm{kg} / \mathrm{day}$ based on the US -ADI, European Commission Standard (ADI) [5].

\section{Conclusion:}

Food contamination is a crucial pathway of human heavy metal contamination. Therefore undertaking risk assessment is important towards increasing life expectancy of the citizens as studies of toxicological effect from experimental animal and sometimes man do not normally agree with health risk assessed on the basis of acceptable scientific criteria.

\section{Reference}

[1]. Sathi D. Balakrishnana .V. (2008); Food technology, Presence of Heavy Metal in Pork Products in Chanan; India, 3rd Ed, Weiaheim, India pp 192-199.

[2]. Judith, R.T. 1994, Copper In Maurice, E.S, James, A.O. and Moshe, S.L, (Ed) Modern Nutrition in Health and Diseases $8^{\text {th }}$ Edn Part 1 pp. $237-240$

[3]. Dudka, S., Miller, P. (1999); Accumulations of potentially toxic elements in plants are their transfer to human food chain. J. Environment Sci. Health, B 34, 681-708.

[4]. Kolle K. Brown T. (2004); 'Environment Health Perspective Recent Development in low level exposure and intellectual impairment in Children, $9^{\text {th }}$ Ed., London, pp 957-994

[5]. Nasiaddine, L. Parent D. Massin (2002); Food Contamination by Metals and Pesticides in the European Union, should we worry?, Recent Articles, Toxicology Letter 127: 29-41.

[6]. Brito, G.C. Diaz, L. Galindo A. Harchsson D Santiago and M.F Garcia (2005) Levels of Metal in canned meat products intermetallic correlations Built Environment contamination. Taxicol, 44(2) 309-316.

[7]. Edwin E.A Dosunmum, M.I. Bassey, F.I., Wilson, C., Umorem, P., (2009). Comparative Assessment of Proximate Composition of Ascopic Acid and Heavy Metal content of Two Species of Garden Egg Parkistan J. of Nutrition 8(8) 582-584

[8]. Daniel, B and A.K. Edward, (1998): Environmental Science, $5^{\text {th }}$ Ed, John Wiley, New York, pp 278-279.

[9]. Aranha, K. (1994): Environment Chemistry. $3^{\text {rd }}$ Ed., New Age International Ltd Publisher, New Delhi, pp $213-219$.

[10]. WHO Principles of Food Safety, Assessment of Food Additives and Containments in Food. Environmental Health criteria.

[11]. Department of Environment, Food and Rural Affairs (DEFRA) (2000); Assessment of Risk of Human Health from Land Contamination An overview of Development of Soil Guideline Values and Related Research, CLR. Bristol, UK.

[12]. Zukowske, J Birivt, M. (2008); Methodological Evaluation of Method for Dietary Heavy Metal Intake Journal of Food Science 73(2): 21-29.

[13]. Ejaz ul Islam xiane Yung Zhen-Li-He and Quisar mohamood (2007); Assessing Potential Dietary Toxicity of Heavy Metals in Selected Vegetables and Food. J of Zhejiang University of Sc. B vol. (8) 1-13

[14]. Onyenuga V.A(1969); Nigeria Food and Feeding Stuffs, their Chemistry and Nutrient value, $3^{\text {rd }}$ Ed. Ibadan University press, Ibadan pp 215-221.

[15]. Mclaughlin M.J Parker D.R and Clarke, J.M. (1999); Metal and Micro nutrients Food \& Safety issues Journal of field Crop Resources, 60; 143-163.

[16]. John H.H. and Jeanne I.R (1994); Food Additives, Contaminants and Natural Toxins in Maurice ES, A.O James, C.L Mostre and Febiger Edu. Modern Nutrition in Health and Diseases 8.Edn, part П page 1597-1598.

[17]. Allen G, Robert A.C. O’Reilly D.S.J. Stewart M.J and James S. (1995); clinical Biochemistry $2^{\text {nd }}$ Edn, Harcourt Brace \& Company Ltd, pp.114-115. 